Proyecciones Journal of Mathematics

Vol. 37, No 2, pp. 223-237, June 2018.

Universidad Católica del Norte

Antofagasta - Chile

\title{
Generalised Closed Sets in Multiset Topological Space
}

\author{
Karishma Shravan \\ Institute of Advanced Study in Science and Tech., India \\ and \\ Binod Chandra Tripathy \\ Tripura University, India \\ Received: May 2017. Accepted : September 2017
}

\begin{abstract}
In this article, we introduce the notion of generalized closed sets and generalized open sets in multiset topological spaces. We investigate their different properties. We have introduced the notion of some separation axioms and discussed some examples.
\end{abstract}

Key Words : Closed set; Open set; Count function; Wilf equivalent pairs; Separation axiom.

AMS Classification : 03E70; 54A05; 54A35; 54C10; 54C60. 


\section{Introduction}

Set theory is the mathematical theory of well-defined collection of objects that are called members, or elements of the set. Every field of mathematics uses or refers to sets in various ways. They play a vital role in formulating more complex mathematical structure, with the limitation that no elements in the set are repeated. But from a practical point of view, there occur many situations where repetition of elements is essential. This led to the introduction of Theory of multisets which was first studied by Blizard [1] in the year 1989. Thus, a multiset is a collection of elements in which certain elements may occur more than once and number of times an element occurs is called its multiplicity. Over the years, the application of multiset is not only limited to philosophy, logic, linguistics, and physics, but a good number of them has been observed in mathematics and computer science, which have led to the formulation of a comprehensive theory of multisets. Multisets have found many significant applications in different fields of mathematics. Multiset is used in graph theory in colorings of graph which was studied by Okamoto et.al. [5]. Venkateswaram [12] discovered a new class of multisetwilf equivalent pairs. It is considered to be the most general multiset Wilf equivalence till date. In the concept of multi-fuzzy soft set was introduced and an application of this in decision making was given by Yang, Tan, and Meng [13]. Girish and John [2], studied in details on the concept of topological space on multiset introduced by them in [1] in the year 2011. In the same paper, the notions of open msets, closed msets, closure, interior, basis, subbasis, continuity and many other related properties in multiset topological space was defined.

Topological spaces have been studied from different aspects such as $b$ closed sets, mixed fuzzy sets, $\gamma$-open sets, $\gamma$-continuous, extension operators by Tripathy and Acharjee [6], Tripathy and Debnath [7], Tripathy and Ray ([8], [9]) Tripathy and Sarma ([10], [11]) and others.

In this paper, we study the notion of generalised closed sets in multiset topological space and its related properties. 


\section{Definitions and Preliminaries}

First, we represent some basic definitions, notations and results those will be used in this article.

Definition 2.1. A domain $X$, is defined as the set of elements from which msets are constructed. The mset space $[X]^{w}$ is the set of all msets whose elements are from $X$ such that no element occurs more than $w$ times.

Throughout this paper, we denote a multiset drawn from the multiset space $[X]^{w}$ by $M$

Definition 2.2. An mset $M$ drawn from the set $X$ is represented by a count function $M$ or $C_{M}: X \longrightarrow N$, where $N$ represents the set of nonnegative integers.

Here $C_{M}(x)$ is the number of occurrences of the element $x$ in the mset $M$ drawn from the set $X=\left\{x_{1}, x_{2}, \ldots \ldots, x_{n}\right\}$ as $M=\left\{m_{1} / x_{1}, m_{2} / x_{2}, \ldots \ldots . m_{n} / x_{n}\right\}$ where $m_{i}$ is the number of occurences of the element $x_{i}, i=1,2, \ldots . n$ in the mset $M$. The elements which are not included in the mset $M$ have zero count.

Remark 2.1. We define some new notions in multiset topological space on the basis of this count function. Whenever $C_{M}(x)=1$ for every $x \in X$ multisets become structurally equivalent to the class of sets. So whatever results and definition we establish when restricted to this condition must be equivalent to some results in classical set theory.

Consider two msets $M$ and $N$ drawn from a set $X$. The following are the operations defined on the msets will be used in this article

- $M=N$ if $C_{M}(x)=C_{N}(x)$ for all $x \in X$.

- $M \subseteq N$ if $C_{M}(x) \leq C_{N}(x)$ for all $x \in X$.

- $P=M \cup N$ if $C_{P}(x)=\max \left\{C_{M}(x), C_{N}(x)\right\}$ for all $x \in X$.

- $P=M \cap N$ if $C_{P}(x)=\min \left\{C_{M}(x), C_{N}(x)\right\}$. 
- $P=M \oplus N$ if $C_{P}(x)=C_{M}(x)+C_{N}(x)$ for all $x \in X$.

- $P=M \ominus N$ if $C_{P}(x)=\max \left\{C_{M}(x)-C_{N}(x), 0\right\}$ for all $x \in X$.

- The mset complement

$M^{c}=Z \ominus M=\left\{C_{M^{c}}(x) / x: C_{M^{c}}(x)=C_{Z}(x)-C_{M}(x), x \in X\right\}$.

Where $\oplus$ and $\ominus$ represent mset addition and mset subtraction respectively.

The following are basic operations under collection of msets. Let $[X]^{w}$ be an mset space with $C_{Z}(x)$ as the multiplicities of $x \varepsilon X$ and $\left\{M_{1}, M_{2}, \ldots ..\right\}$ be a collection of msets drawn from $[X]^{w}$. Then the following operations are possible under arbitrary collections of msets.

1. The Union

$\cup_{i \in I} M_{i}=\left\{C_{\bar{M}}(x) / x: C_{\bar{M}}(x)=\max \left\{C_{M_{i}}(x): i \in I\right\}\right.$, for all $x \in X\}$.

2. The intersection

$\cap_{i \in I} M_{i}=\left\{C_{\underline{M}}(x) / x: C_{\underline{M}}(x)=\min \left\{C_{M_{i}}(x): i \in I\right\}\right.$, for all $x \in X\}$.

3. The mset complement $M^{c}=Z \ominus M=\left\{C_{M^{c}}(x) / x: C_{M^{c}}(x)=C_{Z}(x)-C_{M}(x)\right.$, for all $\left.x \in X\right\}$.

Definition 2.3. Let $M$ be an mset drawn from a set $X$. The support set of $M$ denoted by $M^{*}$ is a subset of $X$ and $M^{*}=\left\{x \in X: C_{M}(x)>0\right\}$.

Definition 2.4. An mset $M$ is said to be an empty set if for all $x \in X$, $C_{M}(x)=0$.

Definition 2.5. Let $X$ be a support set and $[X]^{w}$ be the mset space defined over $X$. Then for any mset $M \in[X]^{w}$, the complement $M^{c}$ of $M$ in $[X]^{w}$ is an element of $[X]^{w}$ such that $C_{M}^{c}=w-C_{M}(x)$ for all $x \in X$.

The following types of submsets of $M$ can be defined from the mset space $[X]^{w}$ on the basis of multiplicity of elements. 
Definition 2.6. A submset $N$ of $M$ is a whole submset of $M$ with each element in $N$ having full multiplicity as in $M$ i.e., $C_{N}(x)=C_{M}(x)$ for every $x \in N$.

Definition 2.7. A submset $N$ of $M$ is a partial whole submset of $M$ is apartial whole submset of $M$ with at least one element in $N$ having full multiplicity as in $M$ i.e., $C_{N}(x)=C_{N}(x)$ for some $x$ in $N$.

Definition 2.8. A submset $N$ of $M$ is a full submset of $M$ if each element in $M$ is an element in $N$ with the same or lesser multiplicity as in $M$ i.e. $C_{N}(x) \leq C_{M}(x)$ for every $x$ in $N$.

Based on the above defined submsets, power submsets were also defined by Girish and John [?]. Let $M \in[X]^{w}$ be an mset.

- The set of all submsets of $M$ denoted by $P(M)$ is called a power mset of $M$.

- The set of all whole submsets of $M$ is called a power whole mset of $M$ and is denoted by $P W(M)$.

- The set of all full submsets of $M$ denoted by $P F(M)$ is called a power full mset of $M$.

The Cantor's power set theorem fails for msets, it is possible to formulate the following reasonable definition of a power mset of $M$ for finite mset $M$ that preserves Cantor's power set theorem.

Definition 2.9. Let $M \in[X]^{w}$ be an mset. The power mset $P(M)$ of $M$ is the set of all submsets of $M$. We have $N \in P(M)$ if and only if $N \subseteq M$. If $N=\emptyset$, then $N \in \in^{1} P(M)$ and if $N \neq \emptyset$, then $N \in \in^{k} P(M)$ where $k=\prod_{z}\left(\begin{array}{c}{[M]_{z}} \\ {[N]_{z}}\end{array}\right)$, the product $\prod_{z}$ is taken over by distinct elements $z$ of the mset $N$ and $\left|[M]_{z}\right|=m$ if and only if $z \in^{m} M$ and $\left|[N]_{z}\right|=n$ if and only if $z \in^{n} N$ then,

$$
\left(\begin{array}{l}
{[M]_{z}} \\
{[N]_{z}}
\end{array}\right)=\left(\begin{array}{l}
m \\
n
\end{array}\right)=\frac{m !}{n !(m-n) !} .
$$


The power set of an mset is the support set of the power mset and is denoted by $P^{*}(M)$.

Note 2.1. Power mset is an mset but its support set is an ordinary set whose elements are msets.

The following is the Multiset Topology defined by Girish and John [2].

Definition 2.10. Let $M \in[X]^{w}$ and $\tau \subseteq P^{*}(M)$. Then $\tau$ is called a multiset topology of $M$ if $\tau$ satisfies the following properties.

1. The mset $M$ and the empty mset $\emptyset$ are in $\tau$.

2. The mset union of elements of any sub-collection of $\tau$ belongs to $\tau$

3. The mset intersection of the elements of any finite sub-collection of $\tau$ belongs to $\tau$.

Mathematically, a multiset topological space is an ordered pair $(M, \tau)$ consisting of an mset $M \in[X]^{w}$ and a multiset topology $\tau \subseteq P^{*}(M)$ on $M$. Multiset topology is abbreviated as $M$-Topology. The elements of $\tau$ are called open msets. The complement of an open mset in an $M$-Topological space is said to be closed mset.

Definition 2.11. Given a submset $A$ of $M$-topological space $M$ in $[X]^{w}$, the interior of $A$ is denoted by $\operatorname{Int}(A)$ and is defined as the mset union of all open msets contained in $A$. i.e $C_{\operatorname{Int}(A)}(x)=\max \left\{C_{G}(x): G \subseteq A\right\}$.

Definition 2.12. Given a submset $A$ of an $M$-topological space $M$ in $[X]^{w}$, the closure of $A$ is defined as as the mset intersection of all closed msets containing $A$ and is denoted by $C l(A)$, i.e $C_{C l(A)}(x)=\min \left\{C_{K}(x)\right.$ : $A \subseteq K\}$.

Definition 2.13. Let $(M, \tau)$ be an $M$-topological space and $N$ is a submset of $M$. The collection $\tau_{N}=\left\{U^{\prime}=U \cap N: U \in \tau\right\}$ is an $M$-topology on $N$, called the subspace $M$-topology. With this $M$-topology, $N$ is called a subspace of $M$ and its open msets consisting of all mset intersections of 
open msets of $M$ with $N$.

Example 2.1. Let $M=\{3 / a, 4 / b, 2 / c, 5 / d\}$ and $\tau=\{\emptyset, M,\{2 / c\},\{2 / a\},\{3 / a, 2 / b\}$, $\{2 / a, 3 / d\},\{2 / a, 2 / c\},\{3 / a, 3 / b, 3 / d\},\{3 / a, 4 / b, 2 / c\},\{2 / a, 2 / c, 3 / d\}\}$ is an $M$-topology on $M$. If $N=\{2 / a, 2 / b, 3 / d\} \subseteq M$, then

$\tau_{N}=\{\emptyset,\{2 / a, 2 / b, 3 / d\},\{2 / a\},\{2 / a, 2 / b\},\{2 / a, 3 / d\}\}$ is an $M$-topology on $N$ and it is the subspace $M$-topology on $N$.

\section{Generalised closed $m$-sets}

Definition 3.1. Let $M$ be a multiset drawn from a set $X$. A submset $M_{1}$ in an $M$-topological space $(M, \tau)$ is said to be a $g$-closed mset if,

$$
C_{c l\left(M_{1}\right)}(x) \leq C_{O_{1}}(x) \text { for all } x \in X,
$$

whenever

$$
C_{M_{1}}(x) \leq C_{O_{1}}(x)
$$

where $O_{1}$ is an open $m$-set.

Example 3.1. Let $X=\{a, b, c, d\}$ and $M=\{3 / a, 2 / b, 4 / c, 6 / d\}$ be an mset in $[X]^{6}$. Then $\tau=\{\emptyset, M,\{2 / a, 1 / b\},\{2 / b, 3 / c\},\{2 / a, 2 / b, 3 / d\}$, $\{2 / a, 2 / b, 3 / c\},\{2 / a, 1 / b, 2 / c, 3 / d\},\{2 / a, 2 / b, 3 / c, 3 / d\},\{1 / b\},\{2 / a\},\{2 / c\}$, $\{1 / b, 2 / c\},\{2 / a, 1 / b, 2 / c\},\{2 / a, 2 / b, 2 / c\},\{2 / b, 2 / c\},\{2 / a, 2 / b, 2 / c, 3 / d\}\}$ is an $M$-topology.

Let $M_{1}=\{1 / a, 1 / c, 2 / d\} \subset M$. We have,

$$
c l\left(M_{1}\right)=\{1 / a, 1 / c, 3 / d\}
$$

Then $C_{c l\left(M_{1}\right)} \leq C_{O_{1}}(x)$,

whenever $C_{M_{1}}(x) \leq C_{O_{1}}(x)$ for all $x \in X$. 
Hence, $M_{1}$ is a $g$-closed mset in the $M$-topological space $(M, \tau)$.

Theorem 3.1. The union of two $g$-closed msets is also a $g$-closed mset.

Proof. Let $M$ and $N$ be $g$-closed msets. Consider an open mset $M^{\prime}$ such that

$$
C_{M \cup N}(x) \leq C_{M^{\prime}}(x)
$$

Then

$C_{M}(x) \leq C_{M^{\prime}}(x)$ and $C_{N}(x) \leq C_{M^{\prime}}(x)$.

Since $M$ and $N$ are $g$-closed mset.

$C_{c l M}(x) \leq C_{M^{\prime}}(x)$ and $C_{c l N}(x) \leq C_{M^{\prime}}(x)$.

$$
\begin{aligned}
\text { Now, } & \\
C_{c l(M \cup N)}(x) & =\max \left\{C_{c l M}(x), C_{c l N}(x)\right\} \\
& \leq \max \left\{C_{M}(x), C_{N}(x)\right\} \\
& =C_{M}(x) .
\end{aligned}
$$

Hence $M \cup N$ is also a $g$-closed mset.

Remark 3.1. The intersection of any two $g$-closed msets may not be a $g$-closed mset.

Example 3.2. Let $M=\{3 / a, 2 / b, 4 / c, 6 / d\}$ and $\tau=\{\emptyset,\{2 / a\}, M\}$ is an $M$-topology on $M$.

Consider the msets $A=\{1 / a, 3 / b\}$ and $B=\{2 / a, 3 / d\}$. Then it can be easily verified that $A$ and $B$ are $g$-closed mset. However

$A \cap B=\{1 / a\}$, which is not a $g$-closed mset.

We state the following result, which can be established using standard technique. 
Theorem 3.2. Let $(M, \tau)$ be an $M$-topological where $M$ is an mset drawn from a set $X$. Suppose $C_{M_{2}}(x) \leq C_{M_{1}}(x) \leq C_{M}(x)$, for every $x \in X, M_{2}$ is a $g$-closed mset relative to $M_{1}$ and that $M_{1}$ is $g$-closed submset of $M$. Then $M_{2}$ is $g$-closed relative to $M$.

Theorem 3.3. Let $M$ be a topological space in $[X]^{w}$. If $N$ is any $g$-closed mset in $M$ and $C_{N}(x) \leq C_{P}(x) \leq C_{c l N}(x)$, then $P$ is $g$-closed.

Proof. Let $O$ be any open mset such that,

$$
C_{P}(x) \leq C_{O}(x)
$$

From the given condition, $C_{N}(x) \leq C_{O}(x)$.

But $N$ is $g$-closed and so we have,

$$
C_{c l N}(x) \leq C_{O}(x)
$$

Since,

$C_{P}(x) \leq C_{c l N}(x)$ we have,

$$
C_{c l P}(x) \leq C_{c l N}(x)[\text { Since } \operatorname{cl}(\operatorname{cl} N)=\operatorname{cl} N]
$$

From (3.2) and (3.3) we have,

$C_{c l P}(x) \leq C_{O}(x)$

Hence $P$ is $g$-closed.

Now we introduce the notion of generalised open msets.

Definition 3.2. An mset $M_{2}$ will be called generalised open mset $(g$-open) if and only if its closure $M_{2}^{c}$ is $g$-closed. 
Theorem 3.4. An mset $M_{2}$ is $g$-open if and only if

$$
C_{F}(x) \leq C_{\text {int }\left(M_{2}\right)}(x) \text { for all } x \in X,
$$

whenever $F$ is a closed mset and $C_{F}(x) \leq C_{M_{2}}(x)$ for all $x \in X$.

Proof. Let $M_{2}$ be a $g$-open mset. Then $M_{2}^{c}$ is $g$-closed.

So,

$$
C_{c l\left(M_{2}^{c}\right)}(x) \leq C_{O}(x) \text { for all } x \in X
$$

whenever

$$
C_{M_{2}^{c}}(x) \leq C_{O}(x) \text { for all } x \in X \text {, where } O \text { is open. }
$$

But, $C_{c l\left(M_{2}^{c}\right)}(x)=C_{\left(i n t M_{2}\right)^{c}}$ for all $x \in X$.

Hence (4) becomes,

$C_{\left(\text {int } M_{2}\right)^{c}} \leq C_{O}(x)$ for all $x \in X$, whenever $C_{M_{2}^{c}}(x) \leq C_{O}(x)$ for all $x \in X$.

By De-Morgan's law,

$C_{O^{c}}(x) \leq C_{\left(\text {int } M_{2}\right)}(x)$, whenever $O^{c}=F$ (Since $O$ is open) is closed and $C_{O^{c}}(x) \leq C_{M_{2}}(x)$.

Conversely, let there be a closed mset $F$ such that the given condition holds. Then, by De-Morgan's law we have,

$$
C_{\left(i n t M_{2}\right)^{c}} \leq C_{F^{c}}(x) \text {, whenever } C_{M_{2}^{c}}(x) \leq C_{F^{c}}(x) \text { for all } x \in X .
$$

We have,

$$
C_{c l\left(M_{2}^{c}\right)} \leq C_{F^{c}}(x) \text { whenever } C_{M_{2}^{c}}(x) \leq C_{F^{c}}(x) \forall x \in X \text { ( } F^{c} \text { is open). }
$$


Hence $M_{2}^{c}$ is $g$-closed, so $M_{2}$ is a $g$-open mset.

Theorem 3.5. Let $C_{\text {int } M} \leq C_{N} \leq C_{M}$ and if $M$ is $g$-open, then $N$ is $g$-open.

Proof. By hypothesis we have,

$$
C_{\text {int } M} \leq C_{N} \leq C_{M}
$$

By De-Morgan's law,

$C_{M^{c}} \leq C_{N^{c}} \leq C_{(i n t M)^{c}}=C_{c l\left(M^{c}\right)}$, since $M$ is $g$-open, $M^{c}$ is $g$-closed.

Hence it follows from Theorem 3.4 that $N^{c}$ is a $g$-closed mset i.e. $N$ is $g$-open.

Next we introduce the notion of separated msets.

Definition 3.3. Two submsets $M_{1}$ and $M_{2}$ of an $M$-topological space $M$ are said to be separated if

(i) $M_{1}$ and $M_{2}$ are disjoint.

(i)Neither contains accumulation point of other. In other words $M_{1}$ and $M_{2}$ are separated if and only if both the following conditions hold:

$C_{M_{1} \cap c l\left(M_{2}\right)}=0$ and $C_{M_{2} \cap c l\left(M_{1}\right)}=0$ for all $x \in X$.

Now we introduce the notion of $M-T_{1 / 2}$ space and study some of its property.

Definition 3.4. A $g$-closed mset $N$ is the whole $g$-closed submset of $M$ with each element in $N$ having full multiplicity as in $M$ i.e.

$C_{N}(x)=C_{M}(x)$ for all $x \in N$. 
Definition 3.5. An $M$ - topological space is called a $M-T_{1 / 2}$ space if and only if every whole $g$-closed mset is a closed mset.

The notions of $M-T_{0}$ space and $M-T_{1}$ space can be defined as in the case of general topology.

Note 3.1. The property of $T_{1 / 2}$ is strict between $T_{0}$ and $T_{1}$ is clear from the following examples.

Example 3.3. To show that a $M$ - topological space is $M-T_{1 / 2}$ but not necessarily $M-T_{1}$, consider

$M=\{2 / a, 3 / b, 2 / c\}$ and $\tau=\{\emptyset, M,\{2 / a\},\{2 / c\},\{2 / a, 2 / c\}\}$ be its $M$ topology.

It is clear that the space space $\tau$ is not $M-T_{1}$. Now,

$N_{1}=\{2 / a, 3 / b\} \subset M$ and $c l N_{1}=\{2 / a, 3 / b\}$.

Hence $N_{1}$ is $g$-closed mset which is also closed.

$N_{2}=\{3 / b, 2 / c\} \subset M$ and $c l N_{2}=\{3 / b, 2 / c\} \subset M$.

Hence $N_{2}$ is $g$-closed which is also closed.

We have $N_{3}=\{2 / a, 2 / c\} \subset\{2 / a, 2 / c\}$ and $c l N_{3}=M$.

Thus $N_{3}$ is not $g$-closed.

Since, here every $g$-closed mset is closed, so it is $M-T_{1 / 2}$ space.

Example 3.4. To show that a $M$ - topological space is $M-T_{o}$ but not necessarily $M-T_{1 / 2}$, consider

$$
M=\{3 / a, 2 / b, 1 / c\} \text { and } \tau=\{\emptyset, M,\{3 / a\},\{3 / a, 1 / c\}\} \text { be its } M \text {-topology. }
$$


It is clear that the space space $\tau$ is $M-T_{o}$. We have,

$$
\{3 / a, 2 / b\} \subset M \text { and } c l\{3 / a, 2 / b\}=M \subset M
$$

hence $\{3 / a, 2 / b\}$ is $g$-closed but not a closed mset. So the space is not $M-T_{1 / 2}$.

\section{Conclusion}

In this article, we have investigated some properties of the notions of generalized closed sets and generalized open sets from multiset topological space point of view. We have also established a relation between separation axioms from multiset point of view. Some examples have been discussed to clarify some situations.

\section{References}

[1] W. D. Blizard: Multiset theory, Notre Dame Jour. Formal Logic,30, pp. 36-66, (1989).

[2] K. P. Girish and S.J. John: Rough Multiset and its Multiset Topology. Lecture Notes in Computer Science, Springer. Berlin, Heidelberg, 6600, pp. 62-80, (2011).

[3] K. P. Girish and S.J. John: On multiset topologies, Theory Appl. Math. Comput. Sci., 2, pp. 37-52, (2012).

[4] K. P. Girish and S.J. John: Multiset topologies induced by multiset relations Inf. Sci., 188, pp. 298-313, (2012).

[5] F. Okamoto, E. Salehi and P. Zhang: On multiset colorings of graphs, Discuss. Math. Graph Theory, 30, pp. 137-153, (2010).

[6] B. C. Tripathy and S. Acharjee: On $(\gamma, \delta)$-Bitopological semi-closed set via topological ideal, Proyecciones Jour. Math., 33 (3), pp. 245-257, (2014). 
[7] B. C. Tripathy and S. Debnath: $\gamma$-open sets and $\gamma$-continuous mappings in fuzzy bitopological spaces, Jour. Intel. Fuzzy Syst., 24 (3), pp. 631-635, (2013).

[8] B. C. Tripathy and G. C. Ray: Weakly continuous functions on mixed fuzzy topological spaces, Acta Sci. Techn., 36 (2), pp. 331-335, (2014).

[9] B. C. Tripathy and G. C. Ray: On $\delta$-continuity in mixed fuzzy topological spaces, Boletim da Sociedade Paranaense de Matemática, 32 (2), pp. 175-187, (2014).

[10] B. C. Tripathy and D. J. Sarma: On weakly b-continuous functions in Bitopological spaces, Acta Sci. Techn., 35 (3), pp. 521-525, (2013).

[11] B. C. Tripathy and D.J. Sarma: Generalized b-closed sets in Ideal bitopological spaces, Proyecciones Jour. Math., 33 (3), pp. 315-324, (2014).

[12] V. Venkateswaram: A new class of multiset wilf equivalent pairs. Discrete Math., 307, pp. 2508-2513, (2007).

[13] Y. Yang, X. Tan and C. Meng: The multi-fuzzy soft set and its application in decision-making, Appl. Math. Model., 37, pp. 4915-4923, (2013).

\section{Karishma Shravan}

Mathematical Sciences Division

Institute of Advanced Study in Science and Technology

Paschim Boragaon; Guwahati - 781035, Assam,

India

e-mail : karishma_math@rediffmail.com

and 


\section{Binod Chandra Tripathy}

Department of Mathematics

Tripura University; Suryamaninagar

Agartala-799022; Tripura,

India

e-mail : tripathybc@rediffmail.com 